\title{
Consistency of pituitary adenomas: Amounts of collagen types I and III and the predictive value of T2WI MRI
}

\author{
PEILIANG LI $^{1-3}$, DAINAN ZHANG ${ }^{1,2}$, SHUNCHANG MA ${ }^{1,2}$, PENG KANG $^{1,2}$, CHUANBAO ZHANG $^{1,2}$, \\ BEIBEI MAO ${ }^{1,2}$, WENJIANLONG ZHOU ${ }^{1,2}$, XI WANG $^{1,2}$, JIAYI PENG $^{1,2}$, LINHAO YUAN $^{1,2}$, \\ YANGYANG WANG $^{1,2}$, JINFU DIAO ${ }^{1,2}$ and WANG JIA ${ }^{1,2}$ \\ ${ }^{1}$ Beijing Neurosurgical Institute, Capital Medical University; ${ }^{2}$ Department of Neurosurgery, \\ Beijing Tiantan Hospital, Capital Medical University, Beijing 100050; ${ }^{3}$ Department of Neurosurgery, \\ Beijing Ditan Hospital, Capital Medical University, Beijing 100015, P.R. China
}

Received May 9, 2020; Accepted April 4, 2021

DOI: $10.3892 / \mathrm{etm} .2021 .10690$

\begin{abstract}
Pituitary adenomas, the most common type of lesion in the sellar region, rank third among all brain tumors, with an incidence of 73-94 cases per 100,000 individuals. Due to its high resolution, MRI is highly efficient in brain imaging and has emerged as the most appropriate method for tumor consistency evaluation. The present study aimed to assess the levels of collagen types I and III in pituitary adenomas with different consistencies and to determine the value of T2-weighted imaging (T2WI) MRI for predicting tumor consistency. A total of 55 patients with pituitary adenomas were divided into the soft and firm tumor groups according to intraoperative tumor consistency. The ratio of the tumor to Pons' signal intensities on T2WI scans was determined. A receiver operating characteristic curve was plotted to assess the specificity and sensitivity of T2WI in predicting tumor consistency. Average optical density (AOD) values for collagen types I $(0.046 \pm 0.008$ vs. $0.052 \pm 0.012, \mathrm{P}=0.033)$ and III $(0.044 \pm 0.008$ vs. $0.050 \pm 0.010, \mathrm{P}=0.016)$ were significantly lower in the soft tumor group compared with those in the firm tumor group. There was no significant difference in the ratio of the tumor to Pons' signal intensities on T2WI scans. The area under the ROC curve was 0.595 $\pm 0.078(\mathrm{P}=0.250)$. The maximum tumor diameter significantly differed between the soft and firm tumor groups $(\mathrm{P}=0.001)$. AOD values for collagen types I and III were significantly correlated with the maximum tumor diameter $(\mathrm{P}<0.001)$. The amounts of collagen types I and III were elevated in firm pituitary tumors compared with the soft ones. The ratio of tumor to Pons' signal intensities on T2WI scans was not able to accurately predict tumor
\end{abstract}

Correspondence to: Dr Wang Jia, Beijing Neurosurgical Institute, Capital Medical University, 119 South 4th Ring West Road, Fengtai, Beijing 100050, P.R. China

E-mail: jwttyy@126.com

Key words: tumor consistency, collagen types I and III, MRI, pituitary adenomas, T2WI consistency. The size of pituitary adenomas may be associated with the expression levels of collagen types I and III.

\section{Introduction}

Pituitary adenoma (PA) is a common benign tumor in neurosurgery that ranks third among brain tumors (1). Pituitary adenomas are overwhelmingly of soft consistency; thus, typical methods of resection with curettage and suction appear to be clinically efficient (2-5). Tumors of the pituitary gland and sellar region account for $15 \%$ of all brain tumors (6-8). The most common tumors are by far pituitary adenomas and benign neuroendocrine neoplasms confined to the sella (9). Early diagnosis of pituitary tumors is highly significant and their proper classification is of paramount importance for treatment and prognostic purposes (10). The initial treatment of pituitary adenomas includes surgery and certain cases may require chemotherapy with somatostatin analogs, cabergoline and/or pegvisomant (11-13). Compression of and invasion to surrounding structures by PA, including the sella, optic chiasm, optic nerve, cavernous sinus, skull base and brain-stem, may result in serious obstruction of the corresponding organ functions. Tumor texture is considered an important factor influencing the rate of surgical resection $(14,15)$.

In surgery, tumor consistency is one of the most important factors that affect surgical difficulty and safety. Therefore, accurately predicting tumor texture may preoperatively guide surgeons in risk assessment and surgical planning (16). The soft and firm consistencies of tumors directly affect the degree of tumor resection $(17,18)$. Previous studies indicated that preoperative MRI signals may predict tumor consistency (18-20). However, tumor consistency remains controversial and deserves further investigation. A previous study demonstrated that low signal intensity on T2-weighted imaging (T2-WI) MRI is correlated with increased collagen content at pathologic diagnosis, and therefore, fibrous consistency (3).

Pierallini et al (5) pointed out that signal intensity on diffusion-weighted imaging (DWI) is associated with the collagen content on pathologic examination. However, other studies 
indicated no statistically significant correlation between consistency and imaging $(1,3,21,22)$.

Of note, collagen types I and III are highly important in pituitary adenomas (21), while differences in collagen composition between soft and firm pituitary tumors have remained largely elusive.

In previous studies on the texture of pituitary tumors, quantitative analysis with immunofluorescence was rarely used for evaluating the collagen content. Furthermore, whether $\mathrm{T} 2 \mathrm{WI}$ is able to predict tumor texture has remained controversial (20). Therefore, the present study aimed to evaluate the levels of collagen types I and III in pituitary adenomas with different consistencies using immunofluorescence. In addition, the significance of T2-weighted imaging (T2WI) sequences in predicting tumor consistency was highlighted.

\section{Materials and methods}

Patients. In the present prospective study, 55 patients with pituitary tumors, who were admitted to Beijing Tiantan Hospital, Capital Medical University (Beijing, China) between September 2018 and March 2019, were recruited. The patients' demographic and clinical data, including sex, age at surgery, clinical subtypes and Knosp grade (23) were recorded. All patients underwent transphenoidal or transcranial surgery. During surgery, the consistency category (soft or firm) was determined by two experienced neurosurgeons with $>20$ years of experience in pituitary tumor surgery. Adenomas that were easily removed in the standard fashion with curettage and suction were considered soft tumors. Those resistant to removal by curettage or requiring adjuvant methods were considered firm tumors. Patients with microadenoma, tumor cysts or stroke were excluded. The study was approved by the Ethics Committee of Beijing Tiantan Hospital, Capital Medical University (Beijing, China) and informed consent was obtained from each patient or their relatives.

Immunofluorescence. Tissue sections (thickness, 4- $\mu \mathrm{m}$ ) were dewaxed in xylene and dehydrated by an ethanol gradient. Citrate buffer ( $\mathrm{pH}$ 6.0) was used for antigen retrieval by boiling for $2 \mathrm{~min}$. After incubation with $0.25 \%$ PBS containing Triton X for 15 min, sheep serum (10\%; ZLI-9021; OriGene Technologies, Inc.) was added for blocking at $37^{\circ} \mathrm{C}$ for $30 \mathrm{~min}$, followed by successive incubations with primary (overnight, $4^{\circ} \mathrm{C}$ ) and secondary (room temperature, $60 \mathrm{~min}$ in the dark) antibodies. Primary antibodies were for COLIII (cat. no. ab23445) and COLI (cat. no. ab90395; both from Abcam). The secondary antibody was goat anti-mouse fluorescein isothiocyanate-labeled $\operatorname{IgG}(1: 100$; cat. no. ZF-0312; OriGene Technologies, Inc.). Furthermore, 4',6-diamidino-2-phenylindole (DAPI) was used for counterstaining. Fluorescence microscopy was used for imaging and microscopic settings were kept stable throughout the imaging. The intensity of green fluorescence signals in each section was measured using Image-J software (v1.52; National Institutes of Health). The contents of collagen types I and III were determined as average optical density (AOD) values. First, fluorescent micrographs were converted into 8-bit grayscale images and inverted to white. Subsequently, measurements (set area, integrated density and limit to the threshold) were set
Table I. Characteristics of the patients with soft and firm tumors.

\begin{tabular}{lrrr}
\hline Item & Soft $(\mathrm{n}=36)$ & Firm $(\mathrm{n}=19)$ & P-value \\
\hline Age (years) & $47.2 \pm 12.6$ & $49.3 \pm 13.5$ & 0.575 \\
Sex & & & 0.024 \\
$\quad$ Male & $15(41.7)$ & $14(72.7)$ & \\
Female & $21(58.3)$ & $5(26.3)$ & \\
Clinical subtype & & & 0.046 \\
$\quad$ Functional & $12(33.3)$ & $1(5.3)$ & \\
Non-functional & $24(66.7)$ & $18(94.7)$ & \\
Hypopituitarism & & & 0.733 \\
Yes & $7(19.4)$ & $5(26.3)$ & \\
No & $29(80.6)$ & $14(72.7)$ & \\
Knosp grade & & & 0.406 \\
0 & $4(11.1)$ & $0(0.0)$ & \\
1 & $4(11.1)$ & $1(5.3)$ & \\
2 & $3(8.3)$ & $4(21.1)$ & \\
3 & $15(41.7)$ & $4(21.1)$ & \\
4 & $10(27.8)$ & $10(52.7)$ & \\
Suprasellar extension & & & 0.057 \\
Yes & $13(36.1)$ & $12(63.2)$ & \\
No & $23(63.9)$ & $7(36.8)$ & \\
\hline
\end{tabular}

Values are expressed as $\mathrm{n}(\%)$ or the mean \pm standard deviation.

via adjusting for thresholds (lower and upper threshold levels were set to 2.71 and 0.01 , respectively). Finally, the integrated density and the sum of the original green fluorescence areas were measured. The AOD was calculated by dividing the integrated optical density (sum) by the area (sum).

MRI. All MRI examinations were performed on a Siemens 3.0 T MRI system (Siemens Healthineers AG). The parameters used for conventional MRI were repetition time of $3000 \mathrm{msec}$ and echo time of $98 \mathrm{msec}$. The OsiriX Lite system (v10.0; Pixmeo Sàrl) was used to analyze MRI data. A region of interest (ROI) was selected within the lesion for homogenous adenomas, while multiple ROIs were selected for heterogeneous lesions and their mean intensity was calculated. An ROI from the pons was selected and the mean intensity was determined. The ratios of tumor to pons signal intensities on T2WI were calculated. The maximum diameter of each tumor was also determined by MRI preoperatively.

Statistical analysis. All statistical analyses were performed using SPSS 25.0 software (IBM Corp.). GraphPad Prism 7 (GraphPad Software, Inc.) was used to plot figures and the receiver operating characteristic (ROC) curve. Continuous variables were expressed as the mean \pm standard deviation or median with interquartile ranges. Categorical variables were expressed as $\mathrm{n}(\%)$. The two-samples t-test or the Mann-Whitney U test was employed to determine significant differences between the two groups for continuous data. Pearson's $\chi^{2}$ and Fisher's exact tests were utilized to assess 
A

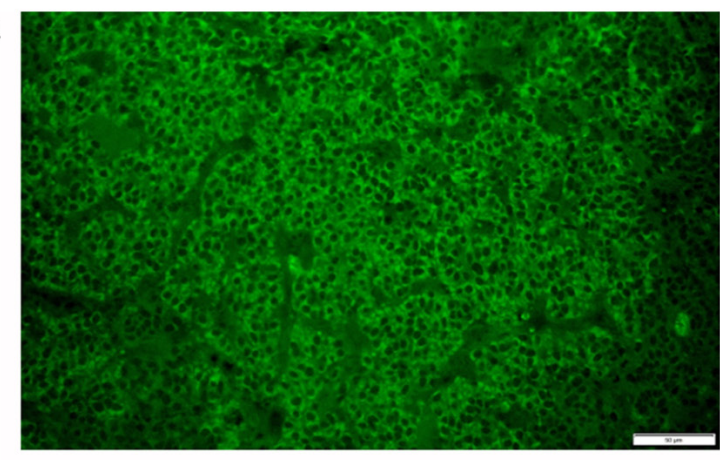

b

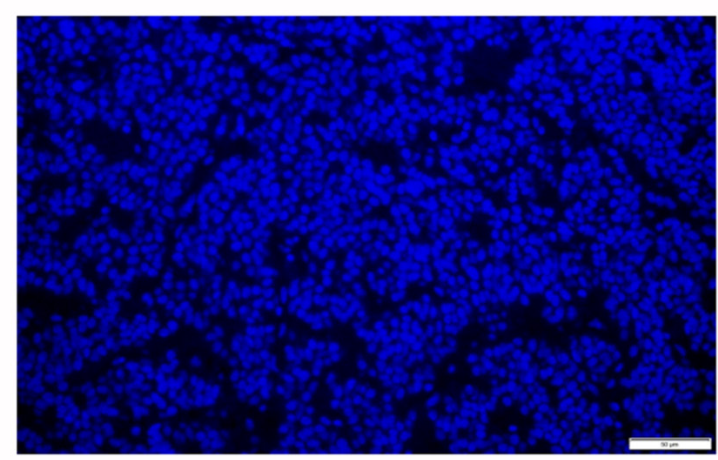

C

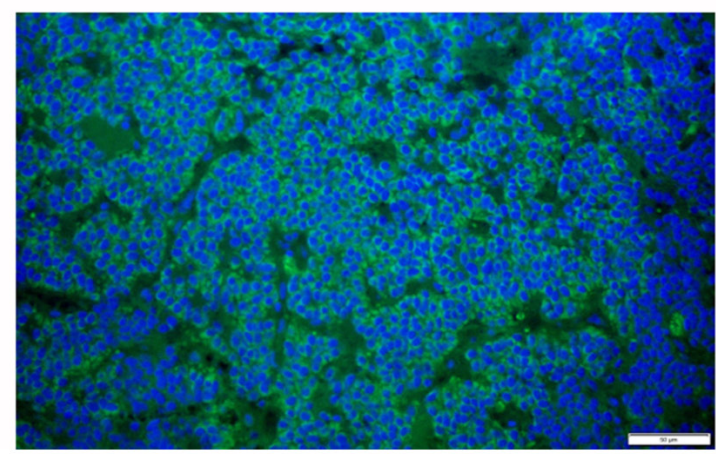

B

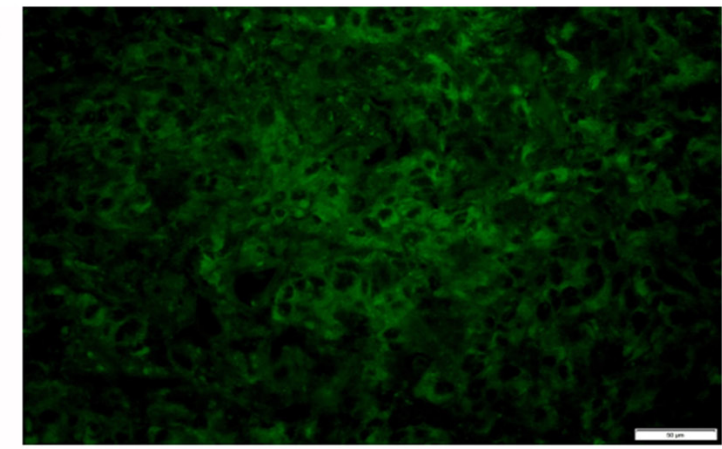

b

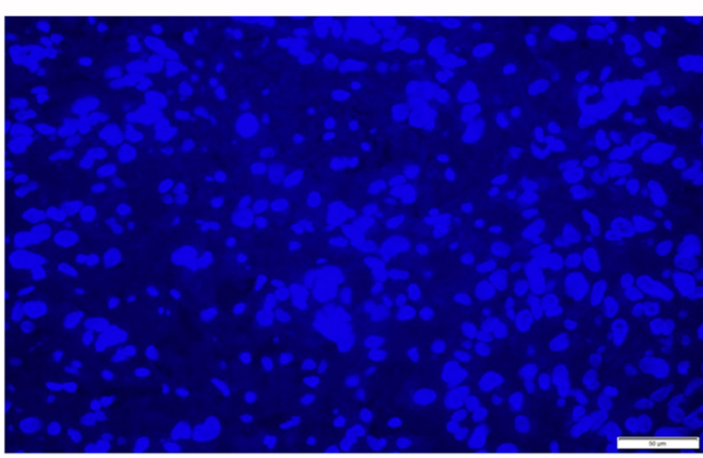

C

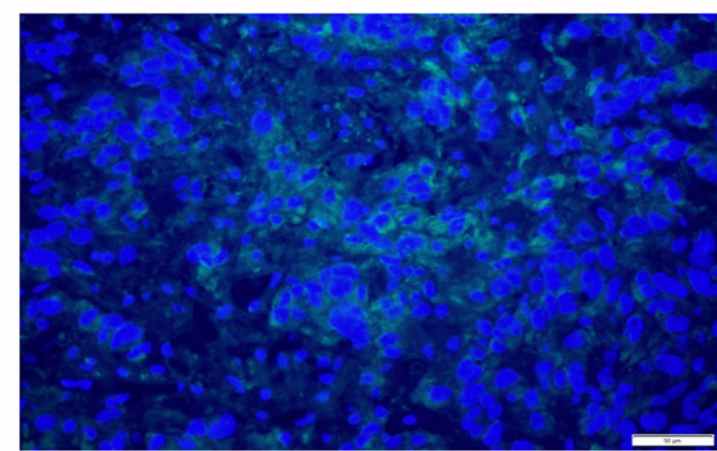

Figure 1. (A) Representative immunofluorescence signals of (A-a) COLI and (A-b) DAPI in firm tumors; (A-c) merged image of A-a and A-b. (B) Representative immunofluorescence signals of (B-a) COLI and (B-b) DAPI in soft tumors; (B-c) merged image of B-a and B-b (magnification, x400; scale bars, $50 \mu \mathrm{m}$ ). COLI, collagen type I.

the differences in proportions between groups. Pearson's correlation analysis was used to investigate the relationship between two individual variables. A two-sided $\mathrm{P}<0.05$ was considered to indicate statistical significance. The ROC curve was plotted to evaluate the significance of signal intensity on T2WI images to predict tumor consistency. An area under the ROC curve (AUC) of $>0.7$ was considered to indicate a certain predictive value.

\section{Results}

Demographics and clinical characteristics. The intraoperative consistency of each tumor was recorded based on resection characteristics and ease of adenoma removal. There were 36 soft and 19 firm tumors and the patients were grouped accordingly. The patients included 29 males and 26 females, with a mean age at diagnosis of $47.9 \pm 12.7$ years (range, 17-72 years). Of the 55 patients, 12 had hypopituitarism prior to surgery. In addition, 39 (71\%) were categorized as Knosp grade 3-4, while 16 (29\%) were grade $0-2$. There were $25(45 \%)$ patients with suprasellar extension, according to MRI findings. The clinical manifestations and preoperative endocrinological examination results indicated that 42 (76\%) cases had non-functional pituitary adenomas. The distribution of soft and firm tumors was different between functional and non-functional pituitary adenomas $(\mathrm{P}=0.046)$. The major clinical manifestations were visual disturbance (38.2\%) and visual field defect (32.7\%). The detailed demographic and clinical features are presented in Table I.

Expression levels of collagen types I and III. The contents of collagen types I (Fig. 1) and III (Fig. 2) were detected using staining in all 55 cases as green signals by immunofluorescence. AOD values for collagen types I $(0.046 \pm 0.008$ vs. $0.052 \pm 0.012, \mathrm{P}=0.033)$ and III $(0.044 \pm 0.008$ vs. $0.050 \pm 0.010$, $\mathrm{P}=0.016)$ were significantly lower in the soft tumor group compared with those in the firm tumor group (Fig. 3). AOD values for collagen types I and III exhibited a significant correlation $(\mathrm{P}<0.001)$, with a correlation coefficient of 0.986 (Fig. 3). 
A

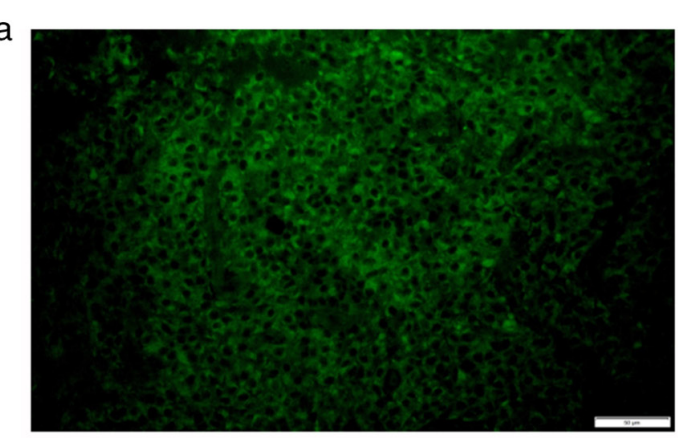

$\mathrm{b}$

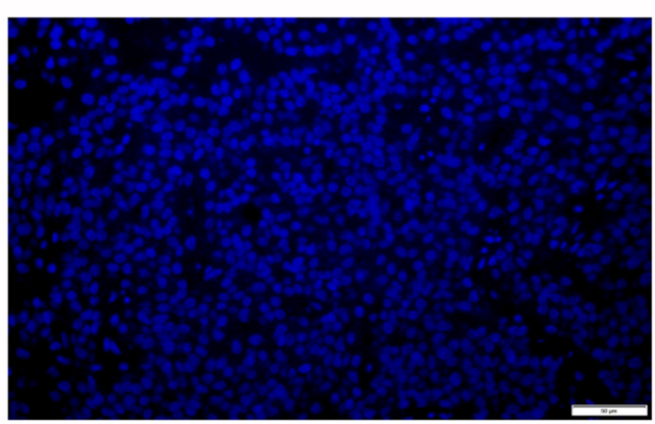

C

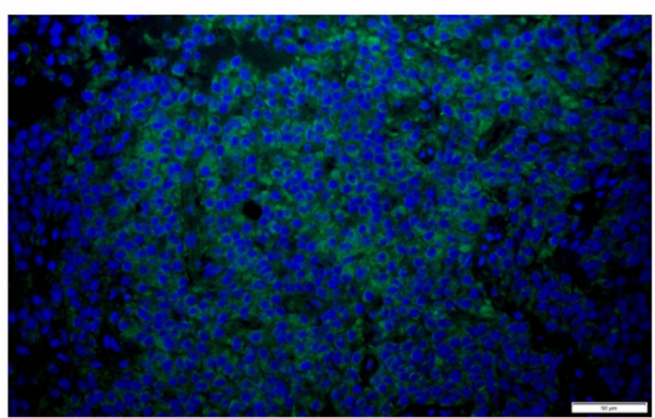

B a

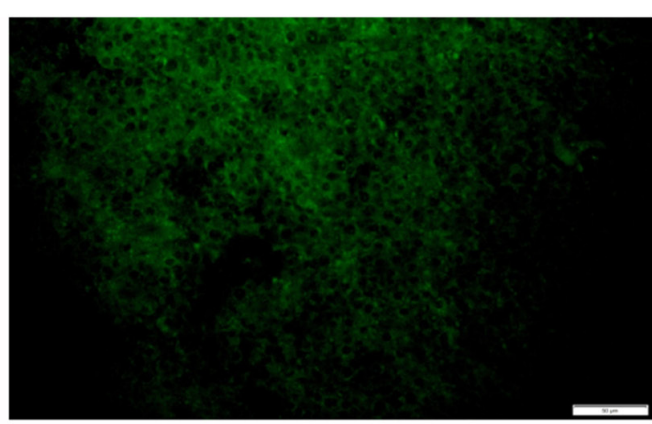

b

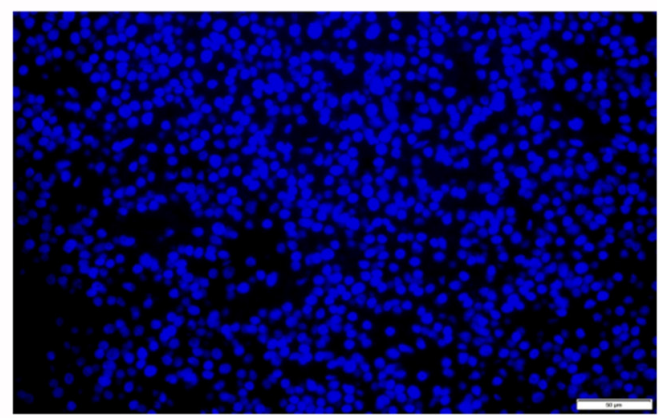

c

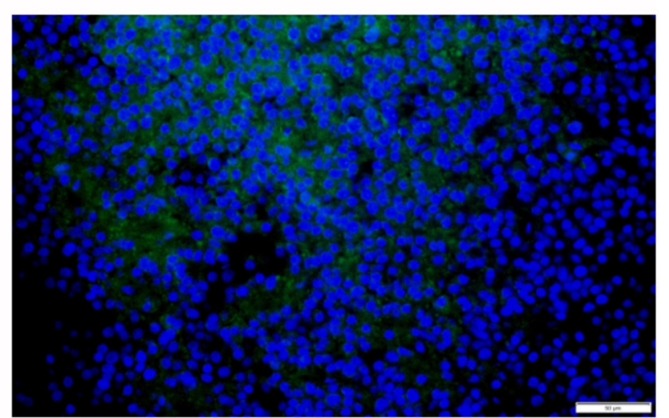

Figure 2. (A) Representative immunofluorescence signals of (A-a) COLIII and (A-b) DAPI in firm tumors; (A-c) merged image of A-a and A-b. (B) Representative immunofluorescence signals of (B-a) COLIII and (B-b) DAPI in soft tumors; (B-c) merged image of B-a and B-b (magnification, $x 400$; scale bars, $50 \mu \mathrm{m}$ ). COLIII, collagen type III.
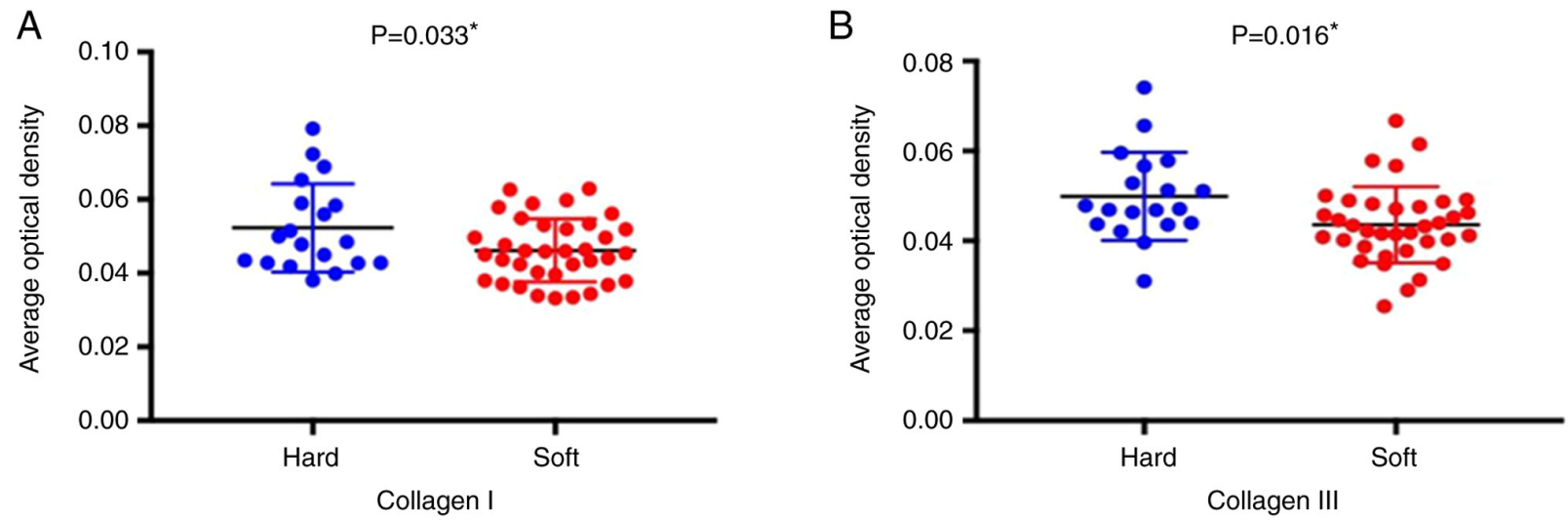

Figure 3. Average optical density values for collagen compared between soft and firm adenomas. (A) Collagen type I (P=0.033) and (B) collagen type III $(\mathrm{P}=0.016)$. Each dot represents one sample. Data are presented as mean \pm standard error of mean. ${ }^{*} \mathrm{P}<0.05$.

T2WI MRI signals are similar in the soft and firm tumor groups. There was no significant difference in the ratio of tumor to Pons' T2WI MRI signal intensities between the soft and firm tumor groups. The ROC curve demonstrated no predictive value for this ratio regarding tumor consistency
[AUC=0.595 (95\% CI: 0.442-0.748); P=0.250; Fig. 4]. The median maximum tumor diameter was $2.82(2.07,3.25) \mathrm{cm}$. There was a statistically significant difference in the maximum tumor diameter between the soft $[2.38(2.01,2.99) \mathrm{cm}]$ and firm $[3.25(2.92,3.68) \mathrm{cm}]$ tumor groups $(\mathrm{P}=0.001$; Table II). 
Table II. Tumor to Pons' signal intensities and tumor diameters in the soft and firm tumor groups.

\begin{tabular}{lcr}
\hline Item & Soft (n=36) & Firm (n=19) \\
\hline Ratio of the tumor to Pons' T2WI MRI signal intensities & $\begin{array}{c}0.53 \pm 0.14 \\
\text { Maximum diameter (cm) }\end{array}$ & $\begin{array}{c}0.55 \pm 0.08 \\
0.38(2.01,2.99)\end{array}$ \\
\hline
\end{tabular}

Values are expressed as the median $(25,75$ th quartiles $)$ or the mean \pm standard deviation. T2WI, T2-weighted imaging.
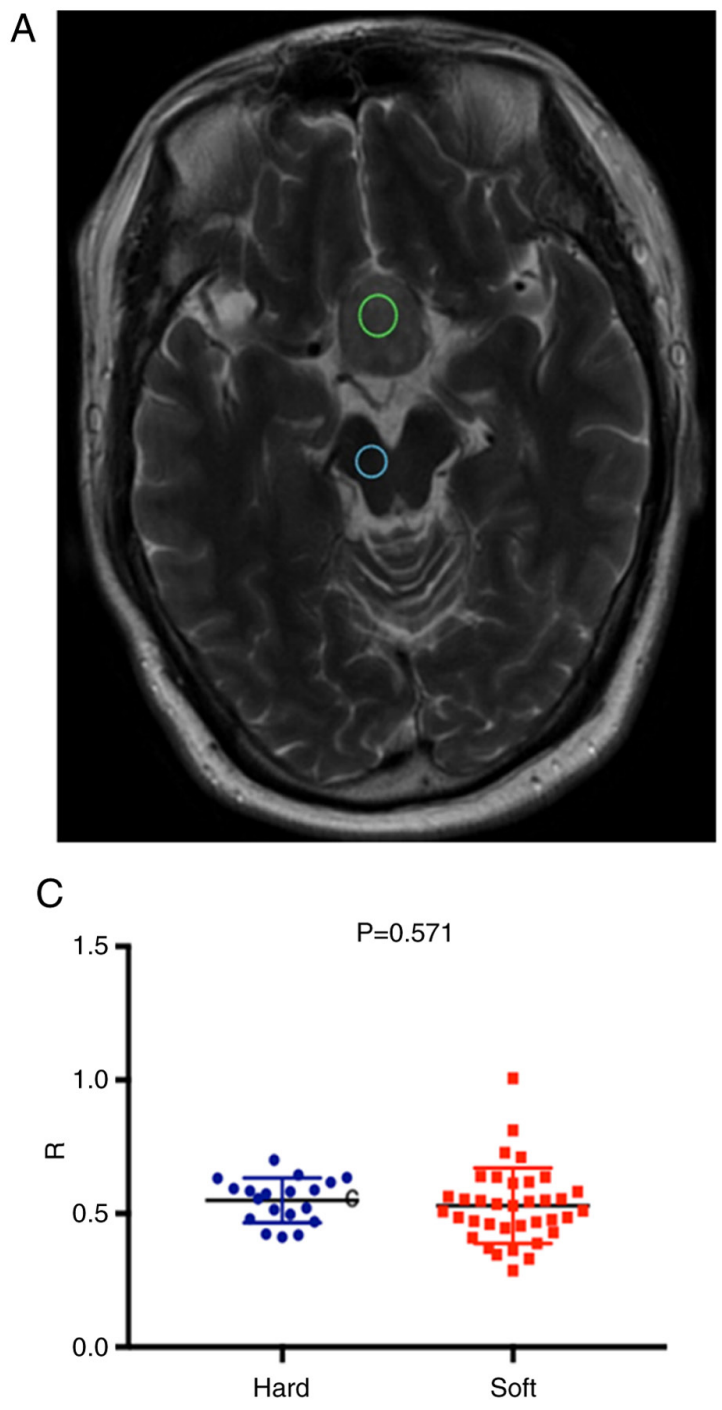

B

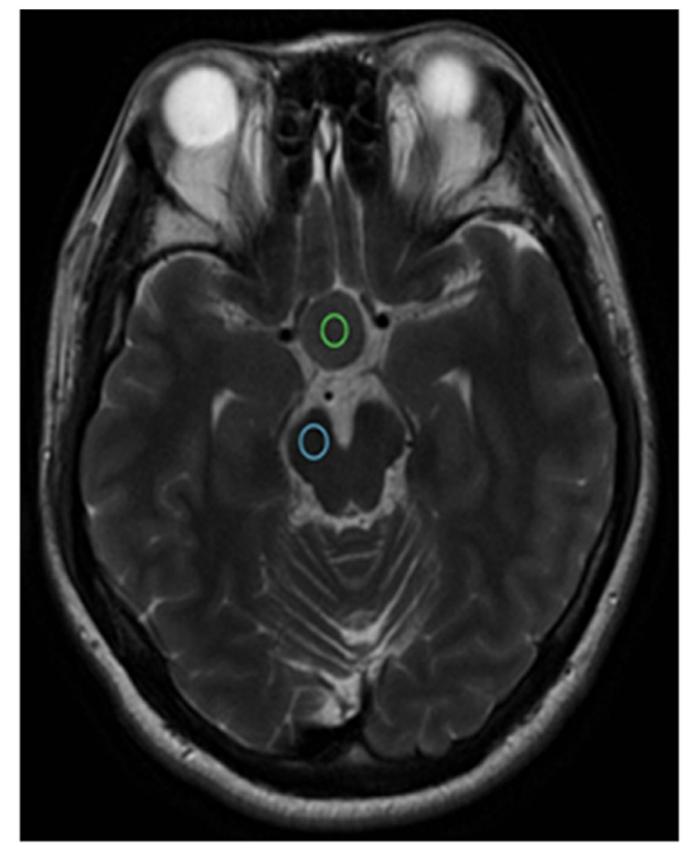

$\mathrm{D}$

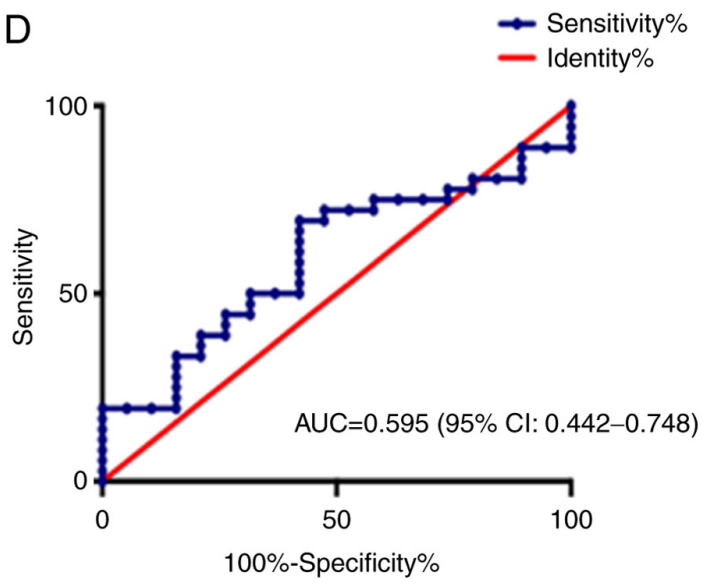

Figure 4. (A and B) Preoperative MRI scans of two patients with pituitary adenomas grouped as having (A) firm and (B) soft tumors during surgery. The regions of interest indicate the tumor (green circles) and Pons (blue circles) signal intensities on T2WI scans. (C) Dot plot of the ratio of tumor to Pons' T2WI MRI signal intensities for soft and firm adenomas $(\mathrm{P}=0.571)$. (D) ROC curve illustrating a lack of predictive value of T2WI MRI signal intensity for tumor consistency $(\mathrm{AUC}=0.595,95 \% \mathrm{CI}$ : 0.442-0.748; $\mathrm{P}=0.250)$. T2WI, T2-weighted imaging; ROC, receiver operating characteristic; AUC, area under the ROC curve; $\mathrm{R}$, ratio.

\section{Discussion}

The present prospective study strongly suggested that the levels of collagen types I and III were higher in the firm tumor group compared with those in the soft tumor group. The size of pituitary adenomas defined by T2WI may be associated with the collagen content, while the ratio of tumor to Pons' signal intensities on T2WI scans were not able to predict tumor consistency.

Previous studies employed immunohistochemistry for determining collagen content in tumors $(3,24,25)$. In the present study, immunofluorescence was applied and ImageJ software was used to analyze the amounts of collagen types I and III. Fibrillar collagen consists of types I, II, III, V and XI. Of these, 
collagen types I, III and V are mainly detected in stromal connective tissues, whereas collagen types II and XI are found in cartilaginous tissues (21).

Type I collagen is the major component of the extracellular matrix in the skin, bone and ligaments, while type III collagen, an important structural protein, is one of the major fibrillar collagens (26). The expression of type III collagen was previously assessed in various benign and malignant tumors by immunostaining (27). In the present study, collagen types I and III were detected in all cases. Collagen types I and III are at times detected in the same fibrils $(28,29)$. Increased expression of collagen type III is observed in several human fibrotic diseases. Collagen type III is known to interact with collagen types I and II in fibril formation and may regulate fibril diameter (27).

The present results suggested that the expression levels of collagen types I and III were higher in firm pituitary adenomas compared with those in soft pituitary adenomas. These results suggest that the collagen content may reflect the firmness of adenomas detected during the operation. According to Pearson correlation analysis, the maximum tumor diameter and collagen content were significantly correlated. Consistent with previous studies $(27,29)$, the present study demonstrated that fibrous tumors may have a larger volume than soft ones. To the best of our knowledge, the secretion and architecture of collagens in tumors are influenced by mutations in tumor suppressor genes (30). Therefore, tumors with high production of collagen may be larger. Various molecules in tumors with high amounts of collagens are associated with cell activity modulation toward more aggressive features (31-33). Different types of cells, including immune cells and fibroblasts, are also present within tumors and participate in the tumor's immune escape and mutual feedback loops that may lead to cancer recurrence $(34,35)$. High collagen content in breast cancer was reported to suppress the proliferation of $\mathrm{T}$ cells (36). Therefore, tumors with high amounts of collagens are not only larger and firmer compared with adenomas, but also more likely to have aggressive features. This should be considered in patient management on an individual basis. Importantly, firm but small tumors may be easier to resect, as they may be limited to the gland.

MRI T2WI signals have a significant role in predicting tumor consistency prior to surgery. Although T2WI was demonstrated to be able to predict the consistency and texture of pituitary adenomas $(3,16,21,37,38)$, opposite results have been previously reported (18-20). In the present prospective study, the ratios of the tumor to Pons' signal intensities on T2WI scans were similar in the firm and soft tumor groups. The present results were consistent with those reported by Thotakura et al (39), who demonstrated that it was not possible to reliably predict the consistency of pituitary adenomas by preoperative MRI. In agreement with this, Mastorakos et al (40) pointed out that the ratio of tumor to cerebellar peduncle T2-WI intensity is not associated with tumor consistency. Furthermore, Bahuleyan et al (2) concluded that the consistency of pituitary macroadenomas was not able to be accurately predicted based on MRI signal intensity. However, MRI sequences may provide more information. DWI may be used for determining the texture of adenomas (16), as well as the Fast Imaging Employing Steady-State Acquisition sequence (41). High-resolution MRI using high-power magnetic fields was also reported to be predictive of texture (42). In future studies, multiple MRI sequences and their associations with collagen content and adenoma consistency require to be assessed. A combination of those sequences may assist in determining the consistency of tumors and performing therapeutic management.

According to the MRI findings of the present study, $63.2 \%$ of patients with firm tumors had suprasellar extension lesions vs. $36.1 \%$ in the soft tumor group. In the firm tumor group, it was difficult to remove the lesions in the suprasellar region. Koktekir et al (43) described the successful use of a transventricular endoscope as an adjunctive measure to remove giant pituitary adenomas by the transsphenoidal approach. They concluded that this technique may be safely used in selected cases. Case selection and surgical strategies should be based on pre-operative MRI findings, ventricular size and the availability of experienced surgeons.

The present study had a number of limitations. First, it was a single-center trial with small sample size, and it was not possible to adjust for important confounders in the statistical analysis, e.g., sex. Thus, larger studies are required to confirm the present findings. In addition, certain tumors had uneven consistency and the specimens may not completely represent the entire tumor. Furthermore, the results indicated that most of the functional pituitary adenomas were soft and most of the non-functional pituitary adenomas were firm. Studies with a larger cohort size are also required to confirm the present results.

In conclusion, the present prospective study demonstrated that the levels of collagen types I and III were higher in pituitary tumors with firm consistency compared with those with soft lesions. In addition, the size of pituitary adenomas observed by MRI was correlated with the amounts of collagen types I and III, and the ratio of tumor to Pons' signal intensities on T2WI scans was not able to predict tumor consistency.

\section{Acknowledgements}

The authors thank Professor Wang Jia from The Department of Neurosurgery of Beijing Tiantan Hospital, Capital Medical University (Beijing, China) for constructive comments and suggestions.

\section{Funding}

The present study was supported by the Capital's Funds for Health Improvement and Research (grant no. CFH2018-1-1071) and National Natural Science Foundation of China (grant no. NSFC82071996).

\section{Availability of data and materials}

The datasets used and/or analyzed during the current study are available from the corresponding author on reasonable request.

\section{Authors' contributions}

PL and DZ supervised the study. PL, WJ and PK designed the experiments. PL, SM, BM and WZ performed the 
experiments. CZ and JD provided new tools and reagents and performed part of data analysis. XW, DZ and JP analyzed the data. PL, YW and LY drafted the manuscript and acquire the clinical data. DZ and WJ revised the manuscript. DZ and CZ confirm the authenticity of all the raw data. All have authors read and approved the final version of the manuscript.

\section{Ethics approval and consent to participate}

The study was approved by The Ethics Committee of Beijing Tiantan Hospital, Capital Medical University (Beijing, China) and informed consent was obtained from each patient or their relatives.

\section{Patient consent for publication}

Not applicable.

\section{Competing interests}

The authors declare that they have no competing interests.

\section{References}

1. Jane JA Jr and Laws ER Jr: The surgical management of pituitary adenomas in a series of 3,093 patients. J Am Coll Surg 193: 651-659, 2001.

2. Bahuleyan B, Raghuram L, Rajshekhar V and Chacko AG: To assess the ability of MRI to predict consistency of pituitary macroadenomas. Br J Neurosurg 20: 324-326, 2006.

3. Iuchi T, Saeki N, Tanaka M, Sunami K and Yamaura A: MRI prediction of fibrous pituitary adenomas. Acta Neurochir (Wien) 140: 779-786, 1998

4. Mahmoud OM, Tominaga A, Amatya VJ, Ohtaki M, Sugiyama K, Sakoguchi T, Kinoshita Y, Takeshima Y, Abe N, Akiyama Y, et al: Role of PROPELLER diffusion-weighted imaging and apparent diffusion coefficient in the evaluation of pituitary adenomas. Eur J Radiol 80: 412-417, 2011.

5. Pierallini A, Caramia F, Falcone C, Tinelli E, Paonessa A Ciddio AB, Fiorelli M, Bianco F, Natalizi S, Ferrante L and Bozzao L: Pituitary macroadenomas: Preoperative evaluation of consistency with diffusion-weighted MR imaging-initial experience. Radiology 239: 223-231, 2006.

6. Sen A, Das C, Mukhopadhyay M, Mukhopadhyay S, Deb S and Mukhopadhyay B: Cytohistological correlation in pituitary tumor and immunological assessment with the help of $\mathrm{Ki}-67$. J Postgrad Med 63: 96-99, 2017.

7. Bidari-Zerehpoosh F, Sharifi G, Novin K and Mortazavi N Invasive growth hormone producing pituitary adenoma with lymphocytic infiltration: A case report and literature review. Iran J Cancer Prev 8: e3504, 2015.

8. Jin G, Hao S, Xie J, Mi R and Liu F: Collision tumors of the sella: Coexistence of pituitary adenoma and craniopharyngioma in the sellar region. World J Surg Oncol 11: 178, 2013.

9. Mete O and Lopes MB: Overview of the 2017 WHO classification of pituitary tumors. Endocr Pathol 28: 228-243, 2017.

10. Lopes MB: Growth hormone-secreting adenomas: Pathology and cell biology. Neurosurg Focus 29: E2, 2010.

11. Molitch ME: Diagnosis and treatment of pituitary adenomas: A review. JAMA 317: 516-524, 2017.

12. Theodros D, Patel M, Ruzevick J, Lim M and Bettegowda C: Pituitary adenomas: Historical perspective, surgical management and future directions. CNS Oncol 4: 411-429, 2015.

13. Dai C, Liu X, Ma W and Wang R: The treatment of refractory pituitary adenomas. Front Endocrinol (Lausanne) 10: 334, 2019.

14. Zhao B, Wei YK, Li GL, Li YN, Yao Y, Kang J, Ma WB, Yang Y and Wang RZ: Extended transsphenoidal approach for pituitary adenomas invading the anterior cranial base, cavernous sinus, and clivus: A single-center experience with 126 consecutive cases. J Neurosurg 112 : $108-117,2010$.
15. Han ZL, He DS, Mao ZG and Wang HJ: Cerebrospinal fluid rhinorrhea following trans-sphenoidal pituitary macroadenoma surgery: Experience from 592 patients. Clin Neurol Neurosurg 110: 570-579, 2008

16. Wei L, Lin SA, Fan K, Xiao D, Hong J and Wang S: Relationship between pituitary adenoma texture and collagen content revealed by comparative study of MRI and pathology analysis. Int J Clin Exp Med 8: 12898-12905, 2015.

17. Zada G, Yashar P, Robison A, Winer J, Khalessi A, Mack WJ and Giannotta SL: A proposed grading system for standardizing tumor consistency of intracranial meningiomas. Neurosurg Focus 35: E1, 2013.

18. Shiroishi MS, Cen SY, Tamrazi B, D'Amore F, Lerner A, King KS, Kim PE, Law M, Hwang DH, Boyko OB and Liu CS: Predicting meningioma consistency on preoperative neuroimaging studies. Neurosurg Clin N Am 27: 145-154, 2016.

19. Tamrazi B, Shiroishi MS and Liu CS: Advanced imaging of intracranial meningiomas. Neurosurg Clin N Am 27: 137-143, 2016.

20. Yao A, Pain M, Balchandani P and Shrivastava RK: Can MRI predict meningioma consistency?: A correlation with tumor pathology and systematic review. Neurosurg Rev 41: 745-753, 2018.

21. Naganuma H, Satoh E and Nukui H: Technical considerations of transsphenoidal removal of fibrous pituitary adenomas and evaluation of collagen content and subtype in the adenomas. Neurol Med Chir (Tokyo) 42: 202-213, 2002.

22. Hagiwara A, Inoue $\mathrm{Y}$, Wakasa $\mathrm{K}$, Haba $\mathrm{T}$, Tashiro $\mathrm{T}$ and Miyamoto T: Comparison of growth hormone-producing and non-growth hormone-producing pituitary adenomas: Imaging characteristics and pathologic correlation. Radiology 228 : 533-538, 2003.

23. Knosp E, Steiner E, Kitz K and Matula C: Pituitary adenomas with invasion of the cavernous sinus space: A magnetic resonance imaging classification compared with surgical findings. Neurosurgery 33: 610-618, 1993

24. Wang H, Li W, Shi D, Ye Z, Qin F, Guo Y and Yuan X: Expression of TGFbeta1 and pituitary adenoma fibrosis. Br J Neurosurg 23: 293-296, 2009.

25. Yiping L, Ji X, Daoying G and Bo Y: Prediction of the consistency of pituitary adenoma: A comparative study on diffusion-weighted imaging and pathological results. J Neuroradiol 43: 186-194, 2016.

26. Ghosh AK: Factors involved in the regulation of type I collagen gene expression: Implication in fibrosis. Exp Biol Med (Maywood) 227: 301-314, 2002.

27. Kuivaniemi H and Tromp G: Type III collagen (COL3A1): Gene and protein structure, tissue distribution, and associated diseases. Gene 707: 151-171, 2019.

28. Fleischmajer R, MacDonald ED, Perlish JS, Burgeson RE and Fisher LW: Dermal collagen fibrils are hybrids of type I and type III collagen molecules. J Struct Biol 105: 162-169, 1990.

29. Cameron GJ, Alberts IL, Laing JH and Wess TJ: Structure of type I and type III heterotypic collagen fibrils: An X-ray diffraction study. J Struct Biol 137: 15-22, 2002.

30. Xu S, Xu H, Wang W, Li S, Li H, Li T, Zhang W, Yu X and Liu L: The role of collagen in cancer: From bench to bedside. J Transl Med 17: 309, 2019.

31. Discher DE, Smith L, Cho S, Colasurdo M, Garcia AJ and Safran S: Matrix Mechanosensing: From scaling concepts in 'Omics data to mechanisms in the nucleus, regeneration, and cancer. Annu Rev Biophys 46: 295-315, 2017.

32. Yamauchi M, Barker TH, Gibbons DL and Kurie JM: The fibrotic tumor stroma. J Clin Invest 128: 16-25, 2018.

33. Oliveira-Ferrer L, Rößler K, Haustein V, Schröder C, Wicklein D, Maltseva D, Khaustova N, Samatov T, Tonevitsky A, Mahner S, et al: c-FOS suppresses ovarian cancer progression by changing adhesion. Br J Cancer 110: 753-763, 2014.

34. Hanahan D and Weinberg RA: Hallmarks of cancer: The next generation. Cell 144: 646-674, 2011.

35. Lambrechts D, Wauters E, Boeckx B, Aibar S, Nittner D, Burton O, Bassez A, Decaluwé H, Pircher A, Van den Eynde K, et al: Phenotype molding of stromal cells in the lung tumor microenvironment. Nat Med 24: 1277-1289, 2018.

36. Kuczek DE, Larsen AMH, Thorseth ML, Carretta M, Kalvisa A, Siersbaek MS, Simões AMC, Roslind A, Engelholm LH, Noessner E, et al: Collagen density regulates the activity of tumor-infiltrating T cells. J Immunother Cancer 7: 68, 2019.

37. Suzuki C, Maeda M, Hori K, Kozuka Y, Sakuma H, Taki W and Takeda K: Apparent diffusion coefficient of pituitary macroadenoma evaluated with line-scan diffusion-weighted imaging. J Neuroradiol 34: 228-235, 2007. 
38. Musleh W, Sonabend AM and Lesniak MS: Role of craniotomy in the management of pituitary adenomas and sellar/parasellar tumors. Expert Rev Anticancer Ther 6 (Suppl 9): S79-S83, 2006.

39. Thotakura AK, Patibandla MR, Panigrahi MK and Mahadevan A: Is it really possible to predict the consistency of a pituitary adenoma preoperatively? Neurochirurgie 63: 453-457, 2017

40. Mastorakos P, Mehta GU, Chatrath A, Moosa S, Lopes MB, Payne SC and Jane JA Jr: Tumor to cerebellar peduncle T2-weighted imaging intensity ratio fails to predict pituitary adenoma consistency. J Neurol Surg B Skull Base 80: 252-257, 2019.

41. Yamamoto J, Kakeda S, Shimajiri S, Takahashi M, Watanabe K, Kai Y, Moriya J, Korogi Y and Nishizawa S: Tumor consistency of pituitary macroadenomas: Predictive analysis on the basis of imaging features with contrast-enhanced 3D FIESTA at 3T. AJNR Am J Neuroradiol 35: 297-303, 2014.
42. Yao A, Rutland JW, Verma G, Banihashemi A, Padormo F, Tsankova NM, Delman BN, Shrivastava RK and Balchandani P: Pituitary adenoma consistency: Direct correlation of ultrahigh field 7T MRI with histopathological analysis. Eur J Radiol 126: 108931, 2020.

43. Koktekir E, Karabagli H and Ozturk K: Simultaneous transsphenoidal and transventricular endoscopic approaches for giant pituitary adenoma with hydrocephalus. J Craniofac Surg 26: e39-e42, 2015.

This work is licensed under a Creative Commons Attribution-NonCommercial-NoDerivatives 4.0 International (CC BY-NC-ND 4.0) License. 